\title{
Ophthalmologic manifestations in Acute Leukemia
}

\author{
Franco Benvenuto*, Mariana Sgroi, Soledad Guillen, Guillermo Chantada and Adriana Fandiño \\ Hospital Nacional de Pediatria J.P.Garrahan, Buenos Aires, CABA, Argentina
}

\begin{abstract}
Objective: To evaluate ophthalmological findings in patients with acute myeloid leukemia and acute lymphoblastic leukemia.

Material and method: A single-center, retrospective, descriptive, observational analysis was conducted of patients with acute myeloid and acute lymphoblastic leukemia seen at the Department of Ophthalmology of Hospital de Pediatría Dr. Prof. Juan P. Garrahanin Buenos Aires, Argentina between March 1, 2017 and February 28, 2018.

Results: Overall, 137 patients with acute leukemia who underwent ophthalmologic examination between March 1, 2017 and February 28,2018 were included. Ages ranged from 0 to 18 years, with a mean of 7.9 years. Remarkably, 22.62\%, i.e. more than one out of every five patients, of the cohort of patients with acute leukemia seen at our center and evaluated at the Department of Ophthalmology had some type of ocular manifestation.
\end{abstract}

Conclusion: Our results show that ocular involvement occurs in a high percentage of patients with leukemia with a clear clinical, humoral, and prognostic correlation, suggesting that ophthalmologic evaluation should be routinely performed in these patients.

\section{Introduction}

Leukemia is a group of diseases that start in the blood-forming tissue, such as the bone marrow, and cause large numbers of abnormal blood cells to be produced and enter the blood stream, sometimes leading to systemic infiltration of immature neoplastic lymphocytes in organs, tissues, and peripheral blood. Ocular involvement in leukemia is well known and all the tissues that compose the eye may be compromised [1-3].

In leukemia, ocular involvement may precede the diagnosis or develop in the course of the disease. The ophthalmologic symptoms may be the result of primary involvement due to infiltration of the ocular tissues or be secondary to hematological changes or changes in hemostasis produced by the disease $[2,4,5]$.

In primary leukemic infiltration three different patterns are recognized: Uveal infiltration of the anterior segment; infiltration of the orbit, including chloromas, orbital hemorrhages, and proptosis; and a neuro-ophthalmologic pattern, characterized by central nervous system (CNS) involvement, including optic nerve infiltration, cranial nerve paralysis, and papilledema [1,4,6,7].

Secondary findings, such as vitreo-retinal hemorrhages, glaucoma, infections, and alterations of the ocular surface, may be due to hematological abnormalities resulting from the presence of anemia, thrombocytopenia, and orhyperviscosity as well as adverse effects of treatments with corticosteroids, chemotherapy, bone marrow transplantation, and radiotherapy; and lastly, to immunosuppression caused by the sum of several of the aforementioned factors [1-10].

In the past, it was believed that eye manifestations did not modify the prognosis of the disease. However, over the years, several studies have shown that the presence of ocular involvement is associated with a poor prognosis in acute leukemia in childhood. Both in acute myeloid leukemia (AML) and acute lymphoblastic leukemia (ALL), the presence of specific orbital and eye lesions have a high statistically significant correlation with disease relapse or CNS involvement resulting in a lower survival rate. In patients with leukemia, the finding of leukemic infiltrates, among other signs, warrants a systemic and neurological reevaluation. The above data suggest that an ophthalmological evaluation should be performed in all patients with leukemia [8,9,11-13].

Advances in technology and treatment modalities have led to higher survival rates in patients with acute leukemia and have resulted in an increased incidence of ocular manifestations. Relapse of leukemia is often diagnosed after presentation of ocular symptoms. These presentations are often asymptomatic and are incidental findings on routine ophthalmologic examination.

\section{Objective}

Although for patients with a diagnosis of or suspected leukemia a multidisciplinary approach is used, specific ophthalmological follow-up, consisting of visual acuity control, biomicroscopic examination, intraocular pressure measurement, and fundoscopy, is important because of the correlation between the general features and ophthalmology findings.

Ophthalmology findings in patients with AML and ALL seen at the Department of Ophthalmology of Hospital de Pediatría Dr. Prof. Juan P. Garrahanbetween March 1, 2017 and February 28, 2018 were assessed.

${ }^{\star}$ Correspondence to: Franco Benvenuto, Hospital Nacional de Pediatria J.P.Garrahan, Buenos Aires, CABA, Argentina, E-mail: fran_benvenuto@ hotmail.com

Key words: leukemia, retinal hemorrhage, papilledema, pediatrics

Received: January 02, 2020; Accepted: January 20, 2020; Published: January 23 2020 


\section{Material and methods}

A single-center, retrospective, descriptive-observational analysis was conducted including all patients with AML and ALL seen at the Department of Ophthalmology of Hospital de Pediatría Dr. Prof. Juan P. Garrahan between March 1, 2017 and February 28, 2018.

All patients underwent complete ophthalmological examination including evaluation of visual acuity and eye movement, biomicroscopy (slit lamp), and fundoscopy (indirect binocular ophthalmoscope). Imaging studies were also performed.

Patient data were digitally stored in tables especially developed for the protocol (Microsoft Office Excel ${ }^{\circledR}$ ).

The variables age, sex, diagnosis, presence or absence of ophthalmologic findings, type of ophthalmologic findings, considering retinal lesions, papilledema, cataracts, orbital infiltration, proptosis, and involvement of the eye surface, as well as hematologic findings were evaluated in the analysis.

\section{Statistical analysis}

Continuous variables are presented as mean and standard deviation or mean and interquartile range according to their distribution. Categorical variables are presented as percentages. Dependent variables were assessed using multivariate analysis.

\section{Results}

Overall, 137 patients with acute leukemia underwent ophthalmological examination between March 2017 and February 2018. Ages of the patients ranged between 0 and 18 years, with a mean of 7.9 years. 62 patients were female and 75 male.

The largest group consisted of 99 patients with a diagnosis of ALL accounting for $72.27 \%$ of the series. Mean age was 8.5 years. The second group included 38 patients with a diagnosis of AML including $27.73 \%$ of the patients. The mean age of the patients in this second group was 6.4 years.

Of all the patients (137), 31 patients had ophthalmologic findings, accounting for $22.62 \%$ of the series. When considering the two subgroups, of 99 patients in the ALL group 22 (22.12\%) and of 38 patients in the AML groupnine $(23.6 \%)$ had ophthalmologic manifestations.

In 22/99 patients with ALL the following ophthalmological manifestations were found: intraretinal and preretinal hemorrhagesin 10 patients, papilledema (optic nerve) in five patients, ocular surface involvement (corneal ulcers and conjuntivitis) in five patients, retinocoroideal infiltrationin one patient, and proptosis in one patient.

In 9 of 38 patients with AML the following ophthalmological manifestations were found: intraretinal and preretinal hemorrhages in four patients, papilledema (optic nerve) in three patients, cataracts in one patient, and proptosis in one patient.

In patients with retinal hemorrhage $(\mathrm{n}=14)$ mean hematological findings were: Hemoglobin 7.66 (6.5-8.5), erythrocytes 2.5 (2.1-3.1), platelets 76 (8-384), and hematocrit 20.8 (20.4-26.8)

\section{Discussion}

A large sample of pediatric patients with lymphoblastic and myeloid-type acute leukemias were studied. In the majority of those who had ocular involvement, the manifestations were secondary to the hemostasis of the patient due to changes in the hematological levels. Involvement of the posterior segment was more common than that of the anterior segment, predominantly in the retina.

Of all the patients with leukemia $(n=137), 106$ did not and 31 patients did have ophthalmological manifestations, the latter accounting for $22.62 \%$ of the sample. These figures are comparable with 35.4\% reported in Malaysia and 39\% reported in the United States but differ from the rates of $69 \%$ and $77.8 \%$ reported in Ethiopia and Nigeria, respectively. This may be related to the lack of human and material resources for the diagnosis and delayed treatment of the disease in these countries [12-15].

In a study of 288 patients with leukemia, Reddy, et al. found that $49.1 \%$ of the adults and only $16.5 \%$ of the children had some type of ocular involvement. Guyer, et al. studied ocular findings only in patients with acute leukemia and found a similar difference of $73.9 \%$ versus $15 \%$ in adults and children, respectively $[16,17]$.

According to the literature, CNS involvement and bone marrow relapse are more common in patients with ALL with ocular manifestations, and therefore ocular involvement is related to a worse prognosis regarding survival $[5,6,9]$.

Russo, et al. studied a series of 180 children with acute leukemia associated with ophthalmologic manifestations and found that these manifestations were more frequent in patients with AML. These authors concluded that relapse of leukemia was more often seen in those patients that had had ophthalmologic findings. In our study we did not observe any significant differences in the rate of ocular involvement between patients with AML and those with ALL $[18,19]$.

Similar to our study,many reports have shown that the most common ocular signs are findings on fundoscopy, mainly in the retina, of which retinal hemorrhage is the most common. The majority of retinal hemorrhages are related to bleeding diathesis secondary to leukemia. Sharma, et al. stated that rheological changes are the main predisposing and causative factor of the ophthalmologic signs and are mainly found in the posterior segment. They reported that at least $69 \%$ of the patients will have retinal manifestations at some point in the course of their disease. We suspect that the statistical difference between these results and ours may be due to the fact that our series consisted of pediatric patients seen at a reference center for oncology-hematology disease where they receive treatments that meet international standards $[4,20-22]$.

In multivariable analysis we found that hemoglobin levels were the most reliable predictive factor among the retinal findings. It was observed that the risk diminishes in patients with levels higher than $7 \mathrm{~g} / \mathrm{L}$, and that it increased in patients with levels in the range of 5.5 to $7 \mathrm{~g} / \mathrm{L}$. A similar phenomenon was observed in the platelet count: when platelet levels were higher than $50,000 / \mathrm{mm}^{3}$, the retinal signs dramatically reduced. Abu el-Asrar, et al. described that low hemoglobin levels are associated with retinal hemorrhages and soft exudates in patients with ALL, and that high leukocyte levels in patients with AML are related with Roth's spot hemorrhages. Robb, et al. reported that leukocyte counts higher than $1,000,000 / \mathrm{mL}$ and high percentages of blasts were related with a high risk of direct retinal infiltration $[23,24]$.

Pediatric ophthalmologists should be aware of the hemorrhagic diathesis status of these patients as vitreal or subhyaloid bleeds often cause sensory deprivation during a fundamental stage of visual development with the subsequent risk of ambliopía. Involvement of 
the optic nerve is secondary to the increased intracranial pressure due to disease related compromise of the CNS as well as direct infiltration by blast cells. Therefore, in patients with these findings the CNS is certainly affected and treatment should be indicated according to the diagnosis and stage of the disease $[2,10,12]$.

Patients may present with diffuse orbital infiltration that may have a hemorrhagic component and evolve with proptosis, edema of the eyelids, ecchymosis, subconjunctival bleeding, and sometimes pain. Optic nerve involvement is more common than the appearance of an orbital mass in ALL [3,10-12].

In AML a tumor type called granulocytic sarcoma or chloroma due to the greenish color of its surface may occur. Chloromas may even appear before the appearance of blasts in peripheral blood [3,1012]. Generally, it is not necessary to take a biopsy of the lesion, except when a relapse is suspected and ocular features are the only disease manifestations at that moment.

The treatment of choice of ocular infiltration is systemic chemotherapy with the type of chemotherapy depending on the cell lineage

\section{Conclusion}

Our study shows a high prevalence of children with acute leukemia at our hospital.The majority of the children had ALL and median age was less than 10 years.

The rate of patients seen at the Department of Ophthalmology with ophthalmologic manifestations was remarkable, as $22.62 \%$ of all the patients were treated, meaning that some type of ocular involvement was found in more than one in each five cases.

When comparing the LMA with the LLA group, incidence of ocular involvement was statistically similar. Retinal hemorrhages were the most commonly found ophthalmologic manifestation accounting for $45 \%$. All the patients with retinal hemorrhages had anemia and thrombocytopenia.

A positive correlation was found between the presence of papilledema and retinochoroid invasion and a poor outcome.

Our results show the importance of an ophthalmological evaluation in all patients with leukemia, as a high rate of ocular manifestations occur in these patients with a clinical, humoral, and prognostic correlation.

\section{References}

1. Catovsky D (1996) Chronic lymphocytic leukemias and other leukemias of mature B and T cells. In: Weatheral DJ, Ledingham JG, Warrel DA (Eds) Oxford Textbook of Medicine Oxford University Press, Oxford, UK pp: 3419-3422.

2. Kincaid MC, Green WR (1983) Ocular and orbital involvement in leukemia. Surv Ophthalmol 27: 211-232. [Crossref]
3. Leonardy NJ, Rupani M, Dent G, Klintworth GK (1990) Analysis of 135 autopsy eyes for ocular involvement in leukemia. Am J Ophthalmol 109: 436-444.

4. Sharma T, Grewal J, Gupta S, Murray PI (2004) Ophthalmic manifestations of acute leukaemias: The ophthalmologist's role. Eye (Lond) 18: 663-672.

5. Talcott KE, Garg RJ, Garg SJ (2016) Ophthalmic manifestations of leukemia. Curr Opin Ophthalmol 27: 545-551. [Crossref]

6. Gordon KB (1999) Ocular manifestations of leukemia. Ophthalmol Clin North Am 12 235-241.

7. Rosenthal AR (1983) Ocular manifestations of leukemia. A review. Ophthalmology 90: 899-905. [Crossref]

8. Singh AD (2003) The prevalence of ocular disease in chronic lymphocytic leukaemia. Eye (Lond) 17: 3-4.

9. Harmon DE (2012) Types of leukemia In: Harmon DE (eds) Leukemia: Current and Emerging Trends in Detection and Treatment, Rosen Publishing Group, New York, USA, pp: 23-30.

10. Rosenthal AR, Egbert PR, Wilbur JR, Probert JC (1974) Leukemic involvement of optic nerve. Trans Pac Coast Oto Ophthal mol Soc Annu Meet 55: 137-158.

11. Stockl FA, Dolmetsch AM, Saornil MA, Font RL, BurnierJr MN (1997) Orbital granulocytic sarcoma. Br J Ophthalmol 81: 1084-1088. [Crossref]

12. Ohkoshi K, Tsiaras WG (1992) Prognostic importance of ophthalmic manifestations in childhood leukemia. Br J Ophthalmol 76: 651-655.

13. Schachat AP, Markiowitz JA, Guyer DR (1989) Ophthalmic manifestations of leukemia. Arch Ophthalmol 107: 697-700.

14. Alemaheyu W, Shamebo M, Bedri A, Mengistu Z (1996) Ocular manifestations of leukemia in Ethiopians. Ethiop Med J 34: 217-224. [Crossref]

15. Eze B, Ibegbulam G, Ocheni S (2010) Ophthalmic manifestations of leukemia in a tertiary hospital population of adult Nigerian Africans. Middle East Afr J Ophthalmol 17: 325-329.

16. Reddy SC, Jackson N, Menon BS (2003) Ocular involvement in leukemia-a study of 288 cases. Ophthalmologica 217: 441-445.

17. Bitirgen G, Belviranli S, Caliskan U (2016) Ophthalmic manifestations in recently diagnosed childhood leukemia. Eur J Ophthalmol 26: 88-91.

18. Russo V, Scott IU, Querques G (2008) Orbital and ocular manifestations in acute childhood leukemia: clinical and statistical analysis of 180 patients. Eur J Opthalmol 18: 619-623. [Crossref]

19. Koshy J, John MJ, Thomas S (2015) Ophthalmic manifestations of acute and chronic leukemias presenting to a tertiary care centre in India. Indian J Ophthalmol 63: 659664 .

20. Orhan B, Malbora B, Akca Bayar S (2016) Ophthalmologic findings in children with leukemia. A single-center study. Turk J Ophthalmol 46: 62e67.

21. Jackson N, Reddy SC, Hishamuddin M, Low HC (1996) Retinal findings in adult leukemia: correlation with leukocytosis. Clin Lab Haematol 18: 105-109.

22. Guyer DR, Schachat AP, Vitale S (1989) Leukemic retinopathy: relationship between fundus lesions and hematologic parameters at diagnosis. Ophthalmology 96: 860-864. [Crossref]

23. Abu el-AsrarAM, al-Momen AK, Kangave D (1996) Correlation of fundus lesions and hematologic findings in leukemic retinopathy. Eur J Ophthalmol 6: 167-172.

24. Robb RM, Ervin LD, Sallan SE (1978) A pathological study of eye involvement in acute leukemia of childhood. Trans Am Ophthalmol Soc 76: 90-101.

Copyright: (C2020 Benvenuto F. This is an open-access article distributed under the terms of the Creative Commons Attribution License, which permits unrestricted use, distribution, and reproduction in any medium, provided the original author and source are credited. 